out a minimum of 100 ablations per year to confirm the uniformity of performance. Patient follow-up data was analysed at staged intervals for one-year post procedure.

Inclusion criteria are: Patients must have had one prior PVI procedure for PeAF, complete follow up data available and all procedures completed using the Carto 3D Mapping System.

Statistical analysis was carried out using SPSS (IBM SPSS Statistic Version 26). A Kaplan Meier graph was generated to evaluate the AF free interval. All continuous variables were

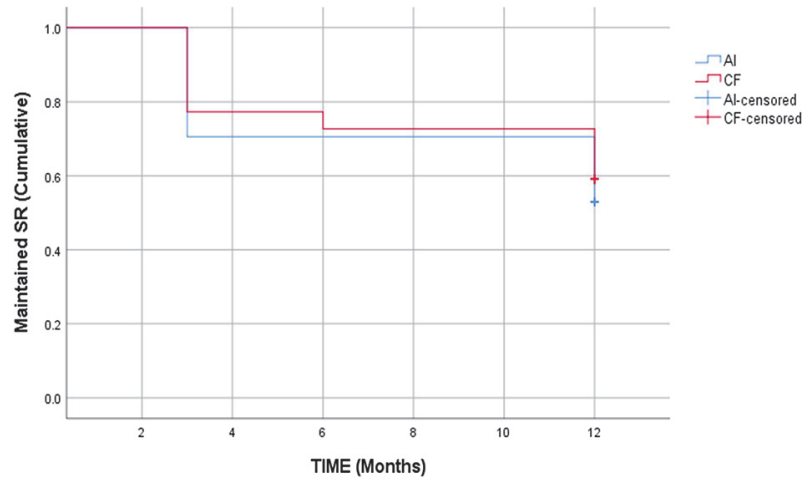

Abstract 6 Figure 1 Kaplien Meier Analysis of AF Free Burden Outcomes over 12 months

Abstract 6 Table 1 Depicts the demographic details, antiarrhythmic medications between $\mathrm{Al}$ group and standard $\mathrm{AF}$ ablation group

\begin{tabular}{|c|c|c|c|}
\hline & $\begin{array}{l}\text { Group } 1(\mathrm{Al}) \\
(\text { mean } \pm \mathrm{SD})\end{array}$ & $\begin{array}{l}\text { Group } 2(C F) \\
(\text { mean } \pm S D)\end{array}$ & $P$ Value \\
\hline $\mathrm{N}$ & 17 & 22 & 0.71 \\
\hline FEMALES (N, \%) & $18 \%$ & $26 \%$ & NS \\
\hline AGE (YEARS) & $62 \pm 10.5$ & $62 \pm 13$ & 0.38 \\
\hline WEIGHT (KG) & $95 \pm 16.8$ & $89.8 \pm 20.9$ & 0.32 \\
\hline Height (Metres) & $1.79 \pm 0.11$ & $1.8 \pm 0.9$ & 0.52 \\
\hline \multicolumn{4}{|l|}{ ATRIAL DATA } \\
\hline AVG DIAMETER (CM) & $3.09 \pm 0.39$ & $3.32 \pm 0.55$ & 0.12 \\
\hline AVG VOLUME (ML) & $4.6 \pm 0.94$ & $4.38 \pm 0.84$ & 0.66 \\
\hline AVG EF (\%) & $58 \pm 11$ & $61 \pm 11$ & 0.85 \\
\hline CHADVASC SCORE & 1.28 & 1.45 & 0.67 \\
\hline PV RECONNECTION & $17 / 17(100 \%)$ & 19/22 (86\%) & 0.1 \\
\hline \multicolumn{4}{|l|}{ ANTI-ARRHYTHMIC } \\
\hline BISOPROLOL & $6 / 17$ & $10 / 22$ & NS \\
\hline SOTOLOL & $7 / 17$ & $8 / 22$ & NS \\
\hline METOPROLOL & $1 / 17$ & $2 / 22$ & NS \\
\hline DRONDARONE & $1 / 17$ & - & NS \\
\hline FLECAINIDE & $3 / 17$ & 2/22 & NS \\
\hline AMIODARONE & $1 / 17$ & $3 / 22$ & NS- \\
\hline VERAPAMIL & $1 / 17$ & - & NS \\
\hline \multicolumn{4}{|c|}{ ANTI ARRHYTHMIC CHANGE } \\
\hline ESCALTION & $2 / 17$ & $7 / 22$ & 0.15 \\
\hline DESCALATION & $11 / 17$ & $6 / 22$ & $0.02^{*}$ \\
\hline NO CHANGE & $4 / 17$ & 9/22 & 0.27 \\
\hline
\end{tabular}

expressed as the mean \pm SD and Students $\mathrm{T}$ Test to was applied to give the significant differences for continuous variables.

Results Patient characteristics are demonstrated in Table 1. There were no significant differences in age, sex, weight, height, CHADVASC or anti-arrythmia agents, which indicated similar patient profile in each cohort. Pulmonary vein reconnections at redo PVI procedure were comparable at $100 \%$ in the AI group and $86 \%$ in the CF group $(\mathrm{p}=0.1)$. Freedom from $\mathrm{AF}$ burden was (mean $8.72 \pm 4.33$ months) in $\mathrm{CF}$ group Versus (mean $9.35 \pm 4.1$ months) in AI guided ablation $(p=0.71)$ (figure 1). The AI group demonstrated greater numbers of patients in whom antiarrhythmic therapy could be deescalated over one year (AI, $n=11 / 17,65 \%$ Vs. CF, $n=2$ / 22 , $9 \%$ with $\mathrm{p}=0.01$ ) while fewer patients underwent escalation of their antiarrhythmic therapy (AI $n=2 / 17,12 \%$ vs $\mathrm{CF}$ $\mathrm{n}=6 / 22,27 \% \mathrm{p}=0.03$ ) (table 1).

Conclusion This is the first study to analyse the outcomes of ablation index on repeat PVI procedures. Despite no significant difference in AF recurrence outcomes demonstrated in this study, there was a significant difference in the medical deescalation in favour of the use of the AI over the short term follow up period. This may reflect effective ablation lesions. A longer-term analysis would be recommended to determine the efficacy of AI use in PeAF redo procedures.

\section{THE CLINICAL UTILITY OF PET-CT IN THE MANGEMENT OF INFECTIVE ENDOCARDITIS}

D Hughes, C Gracias, Z Sharif, BF McAdam. Beaumont Hospital, Dublin, Ireland

\subsection{6/heartinl-2020-ICS.7}

Introduction The latest ESC guidelines on Infective Endocarditis (IE) included the use of PET-CT as an additional diagnostic criterion, particularly for prosthetic valve endocarditis, and several studies showed enhanced sensitivity for the modified Duke criteria. This audit was designed to assess if there was incremental clinical benefit of PET CT imaging in patients suspected of having IE.

Methods A two year retrospective audit was performed on consecutive patients diagnosed with IE, or had a PET-CT for investigation for IE in a large teaching hospital. The modified Duke criteria were used to establish a likelihood of IE and final diagnosis of IE was made based on all clinical information on discharge. The PET-CT scans were classified as having uptake at the site of interest, showed metastatic spread from endocarditis or were negative for either.

Results The audit identified 58 patients with a diagnosis IE or who had a PET-CT for investigation of IE. The mean patient age was 66 years and the in-hospital mortality was 14\%. In all of these cases apart from 1 the final diagnosis was infective endocarditis, either clinically or pathologically (after valve replacement). Of these 11 patients (19\%) had a PET-CT as part of their work up during their inpatient stay. Table 1 provides baseline demographics and clinical details of our cohort. For the patients that had a PET CT the mean age was younger at 55 years compared to 68 years ( $p$ value 0.003 ). As expected, the percentage of patients with prosthetic valves was also higher in the PET CT group at 55\% versus 13\% (P value 0.006). Otherwise there was no significant differences between the two groups. Table 2 outlines the results of the 11 patients who had a PET CT scan as part of their 
diagnostic work up with 7 patients (64\%) having a prosthetic valve (all AVR). All patients had a TOE and this showed a vegetation in 6 patients (50\%), with 4 patients also having an abscess. In 5 cases the scans showed increased FDG activity; 2 scans with peri-aortic valve uptake, 2 scans showing increased aortic mural uptake of unknown significance and one scan showing an embolic phenomenon. In 6 cases there was no increased FDG uptake in the mediastinum/emboli. However, in 5 of these cases the final diagnosis was IE, including 3 who had surgical valve replacement and a pathological diagnosis. There was one case that was a true negative result, resulting in the cessation of antibiotic treatment. Overall, there

Abstract 7 Table 1 Baseline patient characteristics and clinical details

\begin{tabular}{|l|c|c|c|c|}
\hline Detail & PET CT & No PET CT & Total & P Value \\
\hline Patients, no. (\%) & $11(19)$ & $47(81)$ & 58 & - \\
\hline Age, mean (Years) & 55 & 68 & 66 & 0.003 \\
\hline & $6(55)$ & $6(13)$ & $12(21)$ & 0.006 \\
\hline Prosthetic Valve, no. (\%) & $6(100)$ & $3(50)$ & $9(75)$ & - \\
\hline - Aortic valve, no. (\%) & $0(0)$ & $3(50)$ & $3(25)$ & - \\
\hline - Mitral valve, no. (\%) & $2(18)$ & $21(45)$ & $23(40)$ & 0.172 \\
\hline & & & & \\
\hline Device, no. (\%) & $9(82)$ & $36(77)$ & $45(78)$ & 1.000 \\
\hline & $4(36)$ & $17(36)$ & $21(36)$ & - \\
\hline Blood Cultures - Positive, no. (\%) & $1(11)$ & $11(23)$ & $12(21)$ & - \\
\hline - Staph Sp, no. (\%) & $4(36)$ & $8(17)$ & $12(21)$ & - \\
\hline - Strep Sp, no. (\%) & & & & \\
\hline - Other, no. (\%) & $11(100)$ & $47(100)$ & $58(100)$ & 1.000 \\
\hline & $6(50)$ & $38(81)$ & $44(76)$ & 0.110 \\
\hline TOE, no. (\%) & $4(36)$ & $6(13)$ & $9(16 \%)$ & 0.350 \\
\hline - Vegetation, no. (\%) & & & & \\
\hline - Abscess, no. (\%) & $0(0)$ & $8(17)$ & $8(14)$ & 0.331 \\
\hline
\end{tabular}

Abstract 7 Table 2 Details of individual patients who underwent PET CT scanning

\begin{tabular}{|c|c|c|c|c|c|c|c|c|c|}
\hline Patient & $\begin{array}{l}\text { Prosthetic } \\
\text { valve }\end{array}$ & $\begin{array}{l}\text { Blood } \\
\text { cultures }\end{array}$ & TOE & $\begin{array}{l}\text { Abscess } \\
\text { on TOE }\end{array}$ & $\begin{array}{l}\text { Dukes Pre } \\
\text { PET CT }\end{array}$ & PET CT Result & $\begin{array}{l}\text { Discharge } \\
\text { Diagnosis }\end{array}$ & $\begin{array}{l}\text { Valve } \\
\text { replaced }\end{array}$ & $\begin{array}{l}\text { PET CT } \\
\text { Benefit }\end{array}$ \\
\hline$M, 45 \mathrm{Y}$ & $\begin{array}{l}\text { Metallic } \\
\text { AVR } 2007\end{array}$ & MSSA & Positive & No & Definite & $\begin{array}{l}\text { Lung uptake - } \\
\text { possible septic } \\
\text { emboli }\end{array}$ & $\begin{array}{l}\text { Tricuspid Valve } \\
\text { Endocarditis }\end{array}$ & No & True postive \\
\hline$M, 63 Y$ & $\begin{array}{l}\text { Bio AVR } \\
2018\end{array}$ & $\begin{array}{l}\text { Proteus } \\
\text { Mirabilis }\end{array}$ & $\begin{array}{l}\text { Not Definite } \\
\text { IE }\end{array}$ & No & Possible & $\begin{array}{l}\text { Small focus } \\
\text { adjacent to the } \\
\text { aorta. }\end{array}$ & $\begin{array}{l}\text { Prosthetic valve } \\
\text { endocarditis }\end{array}$ & No & True positive \\
\hline$M, 63 Y$ & $\begin{array}{l}\text { Metallic } \\
\text { AVR } 2012\end{array}$ & Staph Capitis & $\begin{array}{l}\text { Not Definite } \\
\text { IE }\end{array}$ & No & Possible & $\begin{array}{l}\text { Mild mural uptake } \\
\text { in ascending Aorta }\end{array}$ & $\begin{array}{l}\text { Prosthetic valve } \\
\text { endocarditis }\end{array}$ & No & False negative \\
\hline$M, 67 \mathrm{Y}$ & $\begin{array}{l}\text { None } \\
\text { (ICD) }\end{array}$ & MSSA & Positive & No & Definite & $\begin{array}{l}\text { FDG avid lung } \\
\text { nodule }\end{array}$ & $\begin{array}{l}\text { Lead } \\
\text { endocarditis }\end{array}$ & N/A & False negative \\
\hline$M, 46 Y$ & None & Strep Mutans & Positive & Yes & Definite & Normal & $\begin{array}{l}\text { Native valve } \\
\text { endocarditis }\end{array}$ & Yes & False negative \\
\hline$M, 29 \mathrm{Y}$ & None & Negative & Positive & Yes & Possible & $\begin{array}{l}\text { Pulmonary } \\
\text { consolidation }\end{array}$ & $\begin{array}{l}\text { Native valve } \\
\text { endocarditis }\end{array}$ & Yes & False negative \\
\hline$M, 84 Y$ & TAVI 2019 & Negative & Negative & No & Negative & Normal & Likely LRTI & No & True negative \\
\hline$M, 66 \mathrm{Y}$ & $\begin{array}{l}\text { Bio AVR } \\
2014\end{array}$ & $\begin{array}{l}\text { Aerococcus } \\
\text { urinae }\end{array}$ & Positive & Yes & Possible & $\begin{array}{l}\text { Uptake at Ao root } \\
\text { possible due to } \\
\text { infection }\end{array}$ & $\begin{array}{l}\text { Prosthetic valve } \\
\text { endocarditis }\end{array}$ & Yes & True postive \\
\hline$M, 39 Y$ & $\begin{array}{l}\text { Metallic } \\
\text { AVR } 2019\end{array}$ & $\begin{array}{l}\text { Enterococcus } \\
\text { Faecalis }\end{array}$ & $\begin{array}{l}\text { Not Definite } \\
\text { IE }\end{array}$ & No & Possible & $\begin{array}{l}\text { Mild Aortic mural } \\
\text { uptake }\end{array}$ & $\begin{array}{l}\text { Prosthetic valve } \\
\text { endocarditis }\end{array}$ & No & False negative \\
\hline$M, 42 \mathrm{Y}$ & None & HACEK & Positive & Yes & Possible & Normal & $\begin{array}{l}\text { Native valve } \\
\text { endocarditis }\end{array}$ & No & False negative \\
\hline$M, 59 \mathrm{Y}$ & $\begin{array}{l}\text { Metallic } \\
\text { AVR } 2016\end{array}$ & $\begin{array}{l}\text { Staph } \\
\text { hominis }\end{array}$ & $\begin{array}{l}\text { Not Definite } \\
\text { IE }\end{array}$ & No & Possible & Normal & $\begin{array}{l}\text { Prosthetic valve } \\
\text { endocarditis }\end{array}$ & Yes & False negative \\
\hline
\end{tabular}


were 3 true positive results, 1 true negative and 7 false negative results.

Conclusion In this study almost 20\% of the cohort underwent PET CT scanning, often in the most clinically challenging and complex cases where the diagnosis is uncertain and guiding surgical referral. Of these only three results were positive for peri-valvular uptake or embolic spread. Two patients had increased uptake in the ascending aorta which was of unknown significance, but was not counted as a positive scan result.

The results in our cohort were not as helpful as reported in other international series. Despite the fact that our numbers were small and used in highly selected cases, they suggest caution in the use of PET-CT in IE. This is particularly pertinent for negative scan results, as 3 of our patients with no cardiac uptake on PET-CT had IE diagnosed pathologically. This highlights the importance of including all the clinical and imaging information in the decision-making algorithm for IE.

\section{INCIDENCE, CLINICAL IMPACT AND PREDICTORS OF THROMBOCYTOPENIA AFTER AORTIC VALVE REPLACEMENT WITH TRANSCATHETER OR SUTURELESS HEART VALVES}

A McInerney, G Triado-Conte, C Hernando Salazar Triviño, A Cruz, P Jimenez-Quevedo, M Carnero, N Gonzalo, J Cobiella, I Nuñez-Gil, H Mejía-Renteria, P Salinas, F Macaya, L Maroto, I Vilacosta, A Fernández-Ortiz, J Escaned, C Macaya, L Nombela-Franco. Hospital Clinico San Carlos, Madrid Spain

\subsection{6/heartjn|-2020-ICS.8}

Background Thrombocytopenia is a poorly understood complication after surgical (mechanical or biological) (SAVR) and transcatheter aortic valve replacement (TAVR). The etiology of this platelet count decrease remains unknown. A higher incidence of thrombocytopenia has been associated with suturelesSAVR (S-SAVR) and transcatheter balloon-expandable valves (BEV) compared to stented and self-expandable valves (SEV), respectively. However, its clinical impact, and furthermore the best cut-off point to predict clinical outcomes has not been elucidated.

Objectives The objective of this study therefore was to analyze the incidence, clinical impact and predictors of thrombocytopenia in patients undergoing transcatheter (TAVR) or S-SAVR replacement.

Methods Consecutive patients $(\mathrm{n}=760)$ with severe aortic stenosis undergoing TAVR $(n=679)$ or S-SAVR $(n=81)$ in a single center between September 2007 and September 2018, and who did not have baseline thrombocytopenia, were included. Patients were classified according to the nadir platelet count, and the time-to-nadir: early nadir ( $<4$ days) or late nadir (greater than or equal to 4 days) post procedure. Receiver Operating Characteristic (ROC) curves for early (30day or in-hospital) mortality were performed using nadir platelet count and percentage decrease in platelet count. Midterm mortality was defined at 2-years. Clinical outcomes were defined according to Valve Academy Research Consortium (VARC-2) criteria.

Results The median percentage decrease in platelet count was $37.8 \%$ [IQR: 28.8-48.4], resulting in moderate $(<100 * 109 /$ L) and severe $(<50 * 109 / \mathrm{L})$ thrombocytopenia in $28.8 \%$ and $4.2 \%$ of patients, respectively. BEV had a lower incidence of moderate-severe thrombocytopenia (27.6\%) compared with SEV (37.7\%, p=0.008) and S-SAVR (51.9\%, p<0.001).
Percentage decrease in platelet count showed a greater area under the curve by ROC analysis than absolute platelet nadir for predicting early mortality with the optimal cut off for percentage platelet decrease being greater than or equal to 46\% (sensitivity: $79.0 \%$ and specificity: 70.6\%). Percentage decrease in platelet count greater than or equal to $46 \%$ predicted early mortality with an odds-ratio of 4.8 (95\% CI: 2.0-11.5). Late nadir platelet was also an independent predictor of early mortality (OR: 4.6, 95\%CI 2.1-10.0). The combination of both factors (greater than or equal to $46 \%$ and greater than or equal to 4 day) predicted higher 2-year mortality $(51.6 \%)$ compared to an early significant nadir (greater than or equal to $46 \%$ and $<4$ day, 24.0\%) and nonsignificant nadir $(<46 \%, 20.2 \%), \quad \mathrm{p}<0.001$ for both comparisons.

Conclusions Moderate-to-severe thrombocytopenia occurred in approximately one third of patients after TAVR or S-SAVR. Percentage decrease in platelet count best predicted short-term clinical outcomes. Only late and significant platelet decrease was associated with mid-term mortality.

\section{LONG TERM OUTCOMES AFTER DEFERRAL OF REVASCULARIZATION FOR INSTENT RESTENOSIS USING PHYSIOLOGICAL ASSESSMENT}

A McInerney, A Travieso Gonzalez, A Castro-Mejia, G Triado-Conte, H Mejía-Renteri, J Escaned, N Gonzalo. Hospital Clinico San Carlos, Madrid Spain

\subsection{6/heartjnl-2020-ICS.9}

Background Instent restenosis (ISR) remains a significant clinical problem. Both bare metal (BMS) and drug eluting stents (DES) can present with ISR, with rates of up to $30 \%$ for BMS and $12 \%$ for second generation DES on routine angiographic surveillance being reported. Current guidelines recommend repeat stenting or the use of drug coated balloons however, treatments can result in multiple stent layers and recurrent stenosis are not uncommon. While abundant data exists on the deferral of non-significant stenosis in native coronary arteries after physiological assessment, there is a paucity of data on the safety of deferral of ISR after physiological assessment.

Objectives To investigate the outcomes of deferred revascularization in patients with non-significant in-stent restenosis (ISR) by physiological assessment.

Methods A propensity-score matched analysis of patients with ISR and native artery disease deferred based on physiological evaluation was performed. Matching was on a 1:2 basis of ISR to native artery disease and data were collected retrospectively. The primary end point was a composite of major adverse cardiovascular events (MACE) including: all-cause mortality, target lesion revascularisation (TLR) or target vessel MI (TVMI) at 36 months in the ISR group versus the matched comparator group

Results A matched cohort of 68 ISR and 136 native artery stenosis were analyzed. The mean percentage stenosis was 53.46 and $54.60 \%$ in the ISR and native artery groups respectively $(p=0.363)$. Most restenosis was within the body of the stent, and of Mehran classification 1C (30 cases, $46.16 \%)$. No significant differences were found between groups regarding the artery interrogated: the left anterior descending artery was most frequently assessed in both groups accounting for approximately half of all physiological 